\title{
Brain Chemistry of Hyper-information, Hyper-thinking and Hyper-consciousness
}

\author{
Aibassov Yerkin, Nakisbekov Narymzhan, Bulenbaev Maxat and Alzhanuly Bakhytzhan \\ Research Institute of New Chemical Technologies and Materials, Kazakh National University Al-Farabi, Almaty 005012, Kazakhstan
}

\begin{abstract}
The authors propose a new approach to the theory of Spin-Boson and Spin-Fermion Topological Model of Consciousness. The authors have studied and proposed new concepts: hyper-information and hyper-thinking and hyper-consciousness.
\end{abstract}

Key words: Hyper-information, hyper-thinking, hyper-consciousness, magnetic field.

\section{Introduction}

The brain is a multilevel multifunctional central nervous system designed to receive, transmit, process (analyze and synthesize) and store information coming from its organs and the environment that regulates and maintains homeostasis in the body, and also promotes and ensures the survival and adaptation of the organism in the constantly changing conditions of exposure to extreme environmental factors. Memory is one of the main functions of the brain.

It is well known that information in human biological fluids, cells and tissues is quantized by atoms and molecules of signal proteins, DNA, mRNA that act as the main regulators of all genetic processes.

One of the most complicated problems of our time is the study of the mechanism of the operation of alpha and gamma rhythms of the brain and the search for their connection with mental activity. The brain carries out a huge number of chemical reactions responsible for the synthesis of memory molecular structures that form memory and the whole system of control of a living organism. The main task of the brain is the conversion of chemical energy into electrical energy (nerve impulses), and neurotransmitters are called to solve this problem.

Corresponding author: Aibassov Yerkin, professor, research field: metal organic chemistry of uranium and thorium, $\mathrm{As}, \mathrm{Sb}$ and $\mathrm{Bi}$.
They control the web of impulses and potentials that guide all the functions of a living organism. In this case, the mechanisms of action of neuroprotectors are chemical.

The purpose of this work is to consider the thermodynamics of the process of hyper-information, hyper-thinking and hyper-consciousness.

\section{Theory}

Thinking - certainly a biological phenomenon, and should therefore be subject to the atomic and molecular description. Science has established that the complex DNA and RNA molecules are capable of performing the function of storage $n$ transmission of information is even more faith in the fact that the complex information processes, including thinking, made atomic-molecular mechanisms.

The fundamental thermodynamic properties of Shannon-type thinking is that the solution to the problem of information is not a process of spontaneous and necessary, ie running with decreasing free energy, but, on the contrary, requires the expenditure of work.

The process of logical thinking is similar to spontaneous thermodynamic process: In both cases, the original particle system (information) to be converted to a finite system of new particles or inferences as spontaneous process in the thermodynamics with decreasing capacity of the free 
energy, and always leads to a more stable state. Spontaneous logical process flows down the free energy and give the thermodynamically stable as a result of the withdrawal or deduction. The process of thinking is described thermodynamically expression:

Work Information: $I_{\text {Inform }}<0, \Delta \varphi_{\text {Inform }}<0$,

The work of judgment (solution): $L_{\text {Solution }} \gg 0$,

$$
\Delta \varphi_{\text {Solution }}>0
$$

where $\Delta \varphi$ - the fall of the free energy in the act of information or discussions.

\section{Results and Discussion}

Bioinformation carriers (DNA, RNA, proteins) provide storage, transportation and various rates of transmission and processing of different volumes of information, which is due to their unequal information capacity and genetically predetermined possibilities of using the information obtained. They are always material. The information transfer rate depends both on the characteristics of the carrier itself and on the characteristics of the information channel. In the blood it is not high, because of the high content of a large number of blood cells. Information on lymphatic pathways is much more rapidly reaching the final destination (other organs and brain) than for example in the circulatory system.

The brain works at very high speeds of receiving and processing information and, accordingly, the removal of unnecessary information from a specific area of the brain must also be very fast, like its arrival. The spent information is removed by transferring it through the venous blood through the kidneys and through the urinary system is removed from the body. Liquor is the brain's pee. Information from all organs should be deleted.

The first information sign is the molecular weight of proteins. On the quantitative basis, most proteins can be ranked by the degree of increase in molecular weight. The second information sign: the localization of protein in the cell. The symptom allows you to determine the amount of protein present in the membrane, the cytoplasm. The third information feature is the protein function in the cell. This feature allows us to describe all the functional processes in which the protein of interest is involved. All these information signs, as well as a number of other information signs are very important for the evaluation of the nerve cell.

Thinking and long-term memory cannot be realized on the pathways of the propagation of nerve impulses through the neural networks of the brain, since the speed of movement of the action potential along the nerve fiber and the time of the synaptic transmission do not provide the really existing speed of the mechanisms of thinking and memory.

The hyperspace theory can become an important milestone of a new era of development and the creation of new strategies for the treatment of neural diseases and the creation of artificial intelligence.

Let's try, for example, to show what hyper-thinking is. Suppose the authors need to solve a complex problem consisting of three stages.

$$
\mathrm{A} \rightarrow \mathrm{B} \rightarrow \mathrm{C} \rightarrow \mathrm{D}
$$

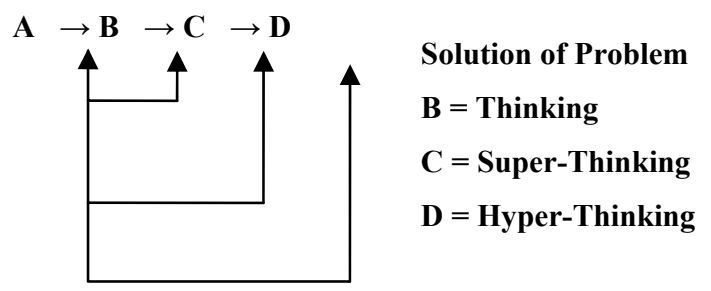

The speed of hyper-thinking is described by Eq. (4):

$$
\mathrm{W}=\mathrm{k}_{1} \mathrm{k}_{2} \mathrm{k}_{3}[\mathrm{~A}][\mathrm{B}][\mathrm{C}][\mathrm{D}] \text {, }
$$

where $\mathrm{k}_{1}, \mathrm{k}_{2}, \mathrm{k}_{3}$ is rate constants of thinking.

How does this show in the chemical example? Suppose it is necessary to get phosphate from phosphine. Usually the reaction goes through three stages.

$$
\begin{array}{r}
\mathrm{PH}_{3} \rightarrow \mathrm{P} \rightarrow \mathrm{HPO}_{3} \rightarrow \mathrm{H}_{3} \mathrm{PO}_{4} \\
\mathrm{P}(-3) \rightarrow \mathrm{P}(0) \rightarrow \mathrm{P}(+3) \rightarrow \mathrm{P}(+5)
\end{array}
$$

To solve this problem, at the first stage the authors oxidize the phosphorus atom P (-3) to obtain elemental phosphorus $\mathrm{P}(0)$. This reaction corresponds to the 
process of thinking. Then, the elemental phosphorus $\mathrm{P}$ (0) is oxidized to the phosphite $\mathrm{P}(+3)$. This reaction corresponds to the process of super-thinking. Then phosphite $\mathrm{P}(+3)$ is oxidized to phosphate $\mathrm{P}(+5)$. This reaction corresponds to the process of hyper-thinking. If phosphate $\mathrm{P}(+5)$ is immediately obtained from phosphine $\mathrm{P}(-3)$, then this corresponds to the process of hyper-thinking.

The human body contains approximately $10^{13}$ cells. The amount of information needed to build such a single structure out of $10^{13}$ ! possible,

$$
\mathrm{I}=\log _{2}\left(10^{13} !\right)=10^{13} \log _{2} 10^{13}=4 \times 10^{14} \text { bit. }
$$

Hence it follows that a decrease in entropy in the construction of the human body from cells will be

$$
\Delta \mathrm{S} \approx 2 \times 10^{-24} \times 4 \times 10^{14} \approx 10^{-9} \mathrm{eu} .
$$

When evaporation of one gram of water, the entropy rises by about $1 \mathrm{eu}$.

The adult body contains about $7 \mathrm{~kg}$ of proteins and $150 \mathrm{~g}$ of DNA, which corresponds to $\approx 3 \times 10^{25}$ amino acid and $\approx 3 \times 10^{23}$ nucleotide residues. To create a single sequence from $20^{3} \times 10^{25}$ possible, for the protein is necessary $\approx 10^{26}$ bit. For DNA it is necessary $\approx 6 \times 10^{23}$ bit. In terms of entropy, the authors obtain 300 and 1.4 eu for proteins and DNA, respectively.

The result of thinking is the output that can be written. They include various elements of the source information.

Thus, the thinking process is modeled on the basis of chemical thermodynamics in the form of spontaneous transitions (Z-1) of varieties of "chances" that are concentrated in one cell in the kth some kinds of a drop of free energy and entropy expressed by the equation of thinking:

The final result of the act of thinking is derivation or inference and in the thermodynamic sense of the word there is a stable state of mind.

The Spin-boson interaction is described by the Eq. (7):

$$
H=-\Delta / 2 \sigma_{\mathrm{x}}+\mathrm{h} / 2 \sigma_{\mathrm{z}}+1 / 2 \sigma_{\mathrm{z}} \Sigma \mathrm{c}_{\mathrm{i}} \mathrm{x}_{\mathrm{i}}+\mathrm{H}_{\mathrm{osc}}
$$

Fermions, unlike bosons, obey Fermi-Dirac statistics: in the same quantum state can be no more than one particle (Pauli exclusion principle).

The spin-fermion interaction is described by the Eq. (8):

$$
\begin{gathered}
\mathrm{H}=\mathrm{J}_{\mathrm{z}} \Sigma \mathrm{S}_{\mathrm{i}} \mathrm{S}_{\mathrm{i}+\varepsilon}+1 / 2 \mathrm{~J}_{\perp} \Sigma\left(\mathrm{S}_{\mathrm{i}}^{+} \mathrm{S}_{\mathrm{i}+\varepsilon}^{-}+\mathrm{S}_{\mathrm{i}}^{-} \mathrm{S}_{\mathrm{i}+\varepsilon}^{+}\right)- \\
\mathrm{t} \Sigma \mathrm{P}_{\mathrm{G}}\left[\mathrm{c}_{\mathrm{i}, \varepsilon} \mathrm{c}_{\mathrm{i}+\varepsilon, \sigma} \exp (\mathrm{i} \varepsilon \cdot \mathrm{k})+\mathrm{c}_{\mathrm{i}+\varepsilon} \mathrm{c}_{\mathrm{i}, \sigma} \exp (-\mathrm{i} \varepsilon \cdot \mathrm{k})\right] \mathrm{P}_{\mathrm{G}}
\end{gathered}
$$

\section{Conclusion}

The authors offer general equations to calculate the hyper-thinking of the work of judgment $L$ and entropy solution $G$ in the presence of a magnetic field:

$$
\begin{gathered}
L=I_{V i n}+\Delta \varphi+1 / 4 \pi H d B \\
G=H_{\text {Sannon }}+\Delta H+1 / 4 \pi H d B
\end{gathered}
$$

The consequence of the above is the ability to save the information entropy and speed of thinking in three dimensions, so the speed of thought is increased by several orders of magnitude.

\section{References}

[1] Shannon, C. E. 1949. "Communication Theory of Secrecy Systems.” Bell Syst. Tech. J. 28 (4): 656-715.

[2] Tony, B., and Barry, B. 1993. The Mind Map Book: How to Use Radiant Thinking to Maximize Your Brain's Untapped Potential. London: BBC Books. 